OPEN ACCESS

Edited by:

Maaike Van Gerwen, Icahn School of Medicine at Mount Sinai, United States

Reviewed by: Paul Horn,

Cincinnati Children's Hospital Medical Center, United States Nalini Selveindran, Hospital Putrajaya, Malaysia

*Correspondence: Ling Qiu

lingqiubj@163.com

${ }^{+}$These authors have contributed equally to this work and share first authorship

Specialty section: This article was submitted to Thyroid Endocrinology, a section of the journal

Frontiers in Endocrinology

Received: 17 November 2021 Accepted: 04 January 2022

Published: 11 February 2022

Citation:

Ma C, Zhong J, Zou Y, Liu Z, Li H,

Pang J, Liu X, Zejipuchi, Tian L, Hou L'a, Wang D, Cheng X and Qiu L (2022) Establishment of Reference Intervals for Thyroid-Associated Hormones Using refineR Algorithm in Chinese Population at High-Altitude Areas.

Front. Endocrinol. 13:816970. doi: 10.3389/fendo.2022.816970

\section{Establishment of Reference Intervals for Thyroid-Associated Hormones Using refineR Algorithm in Chinese Population at High-Altitude Areas}

\author{
Chaochao $\mathrm{Ma}^{1 \dagger}$, Jian Zhong ${ }^{1+}$, Yutong Zou ${ }^{1 \dagger}$, Zhijuan Liu ${ }^{2}$, Honglei $\mathrm{Li}^{1}$, Jinrong Pang ${ }^{2}$, \\ Xiaoxing Liu ${ }^{3}$, Zejipuchi ${ }^{4}$, Liping Tian ${ }^{5}$, Li'an Hou ${ }^{1}$, Danchen Wang ${ }^{1}$, \\ Xinqi Cheng ${ }^{1}$ and Ling Qiu ${ }^{1,6 *}$

\begin{abstract}
${ }^{1}$ Department of Laboratory Medicine, Peking Union Medical College Hospital, Peking Union Medical College \& Chinese Academy of Medical Science, Beijing, China, 2 Department of Clinical Laboratory, People's Hospital of Tibet Autonomous Region, Lhasa, China, ${ }^{3}$ Department of Clinical Laboratory, Ali District People's Hospital, Ali, China, ${ }^{4}$ Department of Clinical Laboratory, Sang Zhu Zi District People's Hospital, Shigatse, China, ${ }^{5}$ Department of Clinical Laboratory, Maternal and Child Health Hospital, Nyingchi, China, ${ }^{6}$ State Key Laboratory of Complex Severe and Rare Diseases, Peking Union Medical College Hospital, Chinese Academy of Medical Science and Peking Union Medical College, Beijing, China
\end{abstract}

Objectives: Diagnosis of thyroid disease among individuals dwelling at high altitude remains a challenge. Reference intervals (RIs) for thyroid-associated hormones among Tibetans living at various high altitudes were established to improve diagnosis.

Methods: One thousand two hundred eighty-one subjects were randomly recruited from Nyingchi, Shigatse/Lhasa, and Ali of Tibet. Thyroid-stimulating hormone (TSH), free triiodothyronine (FT3), and free thyroxine (FT4) were measured by the Cobas e601 electrochemiluminescence analyzer. We used multiple linear regression and variance component analysis to assess the effect of sex, age, and altitude on hormones. Rls were established by refineR algorithm and compared with those provided by the manufacturer.

Results: Serum TSH was significantly lower in males than in females, while FT3 and FT4 were higher in males. Both FT3 and FT4 decreased with increasing age. FT3 increased with altitude, while TSH and FT4 were less influenced by altitude. The RI for TSH was 0.764-5.784 $\mu \mathrm{lU} / \mathrm{ml}$, while for FT4, the Rls were 12.36-19.38 pmol/L in females and 14.84-20.18 pmol/L in males. The Rls for FT3 at Nyingchi, Shigatse/Lhasa, and Ali in females were $4.09-4.98,4.31-5.45$, and $4.82-5.58 \mathrm{pmol} / \mathrm{L}$, while in males, the values were 4.82-5.41, 4.88-5.95, and 5.26-6.06 pmol/L, respectively. The obtained Rls for $\mathrm{TSH}$ and $\mathrm{FT} 4$ were generally higher, while that for $\mathrm{FT} 3$ was narrower than the Rls provided by Cobas.

Conclusions: Specific Rls were established for thyroid-associated hormones among Tibetans, which were significantly different from those provided by the manufacturer.

Keywords: high altitude, reference interval, refineR algorithm, thyrotropin, thyroid hormones 


\section{INTRODUCTION}

Thyroid disease is a prevalent health problem which may lead to potentially devastating healthy consequences (1). In China, the weighted prevalence of overt hyperthyroidism, overt hypothyroidism, subclinical hyperthyroidism, and subclinical hypothyroidism are known to be $0.78 \%, 1.02 \%, 0.44 \%$, and $12.93 \%$, respectively (2). Delayed diagnosis of thyroid disease can lead to adverse effects such as heart failure, atrial fibrillation, and mortality from other cardiovascular diseases, and redundant treatment due to misdiagnosis may also increase disease burden of patients or trigger adverse drug reactions $(1,3,4)$. It was estimated that the rate of overdiagnosis of subclinical hypothyroidism was up to $16 \%$ due to physiological elevation of thyroid-stimulating hormone (TSH) $(5,6)$. Therefore, accurate and timely diagnosis is essential. The diagnosis of thyroid disease is predominantly based on laboratory measurements, of which the accuracy is mainly related to the applicability of reference interval (RIs). For some special groups such as the elderly (7), pregnant women (8), minority, and so on, general RIs may be not applicable. Similarity, habitants in the Tibet Plateau with an average altitude of more than 4,000 m may have their hypothalamic-pituitary-thyroid (HPT) axis altered for adaptation to severe cold, hypobaric hypoxia, sleep disorder, and other unfavorable conditions. This long-term adaptation may lead to thyroid-related hormone levels in Tibetans different from those in the plain (9-11). Even if the effects of sex, age (1214 ), region, sampling time (15), and pregnancy status (16) on thyroid-related hormones are well discussed, however, only few articles $(10,11,17)$ have assessed the influence of altitude on thyroid-associated hormones, let alone establishing altitudespecific RIs.

Currently, direct and indirect methods are the two sampling techniques applied to establish RIs. The direct method is the accepted standard method using a priori or posteriori sampling approach to provide a high internal validity and a minimal bias. However, the process is always costly and time-consuming and has poor feasibility (18). Since it is very difficult to define the apparently healthy individuals for specific groups such as the elderly, pregnant women, and people living in a special environment, the application of the direct method is restricted (18). Conversely, the indirect method with the simple and lowcost performance in a real-world environment utilizes the data mining technique to estimate the healthy distribution from the mixed distribution $(19,20)$. Thus, it can be used for the establishment of RIs when healthy individuals cannot be defined and obtained using direct sampling. The refineR algorithm has been previously proposed for the problem of "data mining" RIs (21). An open-source R code for the establishment of RIs using refineR algorithm has been developed by Ammer (21) and its effectiveness has been also proved. The core of the algorithm is still parameter combination and optimal search. Compared with the forward approach proposed by other indirect algorithms, the refineR algorithm adopts an inverse modeling approach to separate the healthy distribution of observed test results and identifies the optimal model for RI establishment. In short, using indirect methods such as refineR algorithm to establish RIs might be more preferable in Tibetan population, as the chronic exposure to high altitude may induce changes of thyroid-associated hormones for environmental adaptation $(10,11)$.

To help make more accurate clinical decisions on thyroidrelated diseases, we used the variance component model to explore the effects of age, sex, and altitude on thyroidassociated hormones in Tibetan population. Furthermore, we established sex- and altitude-specific RIs for thyroid-related hormones in Tibetans based on the refineR algorithm and evaluated the application.

\section{METHOD AND MATERIAL}

\section{Subjects}

From September 2016 to August 2018, we used a standard questionnaire to recruit participants at Ali (altitude I: 4,2984,352 m), Shigatse/Lhasa (altitude II: 3,670-3,835 m), and Nyingchi (altitude III: about 2,900 m) of Tibet Autonomous Region in China. One thousand two hundred eighty-one indigenous Tibetan subjects were randomly enrolled in our study by the following criteria:

i. Subjects self-report that they are currently in good health and have no major organ system disease,

ii. age $\geq 19$ years,

iii. the subjects were Tibetan,

iv. subjects with no hospitalization in the past 6 months and no illness in the past 4 weeks, and

v. subjects were required to have lived in Tibet for $>1$ year.

\section{Ethical Approval}

This study has been approved by the Ethics Committee of the People's Hospital of Tibet Autonomous Region (Approval No.ME-TBHP-2017-021) and the Ethics Committee of Peking Union Medical College Hospital (Approval No. S-K530). All the subjects had signed the informed consent form.

\section{Sample Collection}

All subjects were requested to maintain on a normal diet and to avert night shifts or strenuous exercise $24 \mathrm{~h}$ before tests. After sitting for 10 to $15 \mathrm{~min}$, fasting venous blood samples of subjects were drawn into red-capped procoagulant-containing Vacuette 5-mL tubes with gel (Greiner Bio-One, Kremsm €unster, Austria) by well-trained nurses and centrifuged at $3,000 \mathrm{rpm}$ for $10 \mathrm{~min}$.

\section{Analytical Performance of Analytes}

The levels of thyroid-related hormones including TSH, FT3, and FT4 were measured using the Cobas e601 electrochemiluminescence analyzer (Roche, Basel, Switzerland) with corresponding reagents, calibrators, and quality controls supplied by the manufacturer. 


\section{Quality Control}

In this study, sample quality was strictly controlled, and all sample collection, processing, and testing personnel were uniformly trained. Serum samples were centrifuged, packaged and frozen within 30 min after sampling, and transported to the Department of Laboratory Medicine of Peking Union Medical College Hospital for unified measurement through a cold chain transportation system with strict temperature control. For all test items, two levels of quality control were implemented before and after each batch test. Samples can be tested only after the quality control is qualified. In addition, the Department of Clinical Laboratory of Peking Union Medical College Hospital is accredited by both ISO15189 and CAP. All the above test items were evaluated by the National Health and Health Commission, and the results were all qualified.

\section{Data Cleaning and Statistical Analysis}

Data cleaning and statistical analysis were performed using $\mathrm{R}$ programming language (V.4.0.5) and MedCalc Statistical software 18.116.6 (Mariakerke, Belgium). Shapiro-Wilk tests and frequency distribution histograms were used to describe the distributions of the items. If the variables satisfy a normal distribution, data were described as mean \pm standard deviations and the Tukey method was used to identify outliers, whereas data rejecting the normality hypothesis were described as medians with quartiles. The Box-Cox method was applied to improve the normality of data before using the Tukey method to identify the outliers. Furthermore, when establishing reference intervals, the Box-Cox was performed again. The standardized regression coefficients of sex, altitude, and age were calculated by multiple linear regression. The variance component model was used to calculate the variation of thyroid- related hormones in gender, age, and altitude, that is, standard deviation or coefficient of variation. The residual standard deviation in the variance component model represents individual variation of thyroid-related hormones. Furthermore, standard deviation ratio (SDR) was expressed as (SDsex, SDaltitude, SDage)/SDresidual and was employed to judge whether the RI of thyroid-related hormones needs to be divided into several partitioning by sex, age, or altitude, and 0.4 is often used as a judgment threshold for thyroidrelated hormones.

The refineR algorithm (21) was implemented using refineR package (version 1.0.0) for the aim of establishing RIs for thyroidassociated hormones. Two-sided $P<0.05$ was considered statistically significant.

\section{RESULTS}

\section{Baseline Information of Participants Enrolled in the Study}

In total, 1,281 participants were enrolled in our study (Nyingchi: $n=363$; Shigatse/Lhasa: $n=473$; Ali: $n=445)$. Females accounted for $65.8 \%, 55.0 \%$, and $51.9 \%$ of the recruits at altitudes III, II, and I, respectively. The median age of the Tibetan population at altitudes III, II, and I was 42, 42, and 34 years, respectively. The results of TPO-Ab and TG-Ab measured by Cobas are shown in Table $\mathbf{1}$.

\section{Effect of Sex, Altitude, and Age on Thyroid-Associated Hormones}

Source variations of each thyroid-related hormone were analyzed as shown in Tables 2, 3. Sex has apparently effects on TSH, FT4, and FT3, showing that females have higher TSH levels but lower FT4 and FT3 levels than males. As shown in Figure 1, both FT3

TABLE 1 | Baseline information of individuals enrolled in this study.

\begin{tabular}{|c|c|c|c|}
\hline Index & Altitude III & Altitude II & Altitude I \\
\hline$n$ & 363 & 473 & 445 \\
\hline Age (years) & $42(32,51)$ & $42(32,52)$ & $34(28,43)$ \\
\hline Sex (female\%) & $65.8 \%$ & $55.0 \%$ & $51.9 \%$ \\
\hline TPO-Ab (IU/L) & $13.25(11.15,16.21)$ & $12.52(10.02,18.46)$ & $14.51(11.45,9.28)$ \\
\hline TG-Ab (IU/L) & $10.00(10.00,10.54)$ & $10.45(10.00,14.28)$ & $14.00(11.23,18.97)$ \\
\hline
\end{tabular}

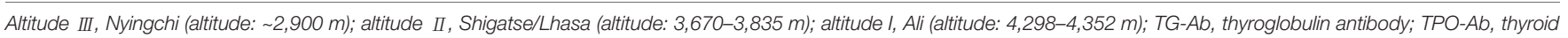
peroxidase antibody.

TABLE 2 | Effect of sex, altitude, and age on thyroid-associated hormones.

\begin{tabular}{|c|c|c|c|c|c|c|c|c|c|c|c|c|}
\hline \multirow[t]{3}{*}{ Index } & \multicolumn{2}{|c|}{ Sex } & \multicolumn{4}{|c|}{ Altitude } & \multicolumn{6}{|c|}{ Age } \\
\hline & \multirow[t]{2}{*}{$\beta$} & \multirow[t]{2}{*}{$P$} & \multicolumn{2}{|c|}{ Al 1} & \multicolumn{2}{|c|}{ Al 2} & \multicolumn{2}{|c|}{ A1 } & \multicolumn{2}{|c|}{ A2 } & \multicolumn{2}{|c|}{ A3 } \\
\hline & & & $\beta$ & $P$ & $B$ & $P$ & $\beta$ & $P$ & $\beta$ & $P$ & $B$ & $P$ \\
\hline TSH & -0.060 & 0.038 & 0.077 & 0.026 & -0.027 & 0.446 & 0.041 & 0.253 & 0.093 & 0.009 & 0.151 & $<0.001$ \\
\hline FT3 & 0.425 & $<0.001$ & 0.164 & $<0.001$ & 0.309 & $<0.001$ & -0.185 & $<0.001$ & -0.257 & $<0.001$ & -0.250 & $<0.001$ \\
\hline FT4 & 0.300 & $<0.001$ & 0.000 & 0.991 & 0.076 & 0.020 & -0.060 & 0.075 & -0.216 & $<0.001$ & -0.235 & $<0.001$ \\
\hline
\end{tabular}

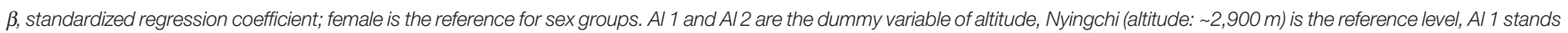

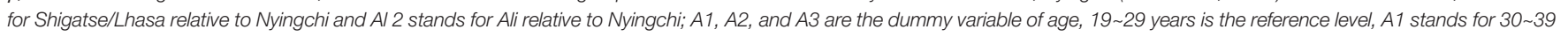
years relative to 19 29 years, A2 stands for 40 49 years relative to 19 29 years, A3 stands for age 50 or older relative to 19 29 years. 
TABLE 3 | Results of variance component model for thyroid-associated hormones.

\begin{tabular}{|c|c|c|c|c|c|c|c|}
\hline \multirow[t]{2}{*}{ Index } & \multirow[t]{2}{*}{ SDRresi } & \multicolumn{2}{|c|}{ Sex } & \multicolumn{2}{|c|}{ Altitude } & \multicolumn{2}{|c|}{ Age } \\
\hline & & SD & SDR & SD & SDR & SD & SDR \\
\hline TSH & 2.18 & 0.33 & 0.2 & 0.00 & 0.0 & 0.34 & 0.2 \\
\hline FT3 & 0.50 & 0.38 & 0.8 & 0.21 & 0.4 & 0.17 & 0.3 \\
\hline FT4 & 2.14 & 0.96 & 0.4 & 0.23 & 0.1 & 0.71 & 0.3 \\
\hline
\end{tabular}

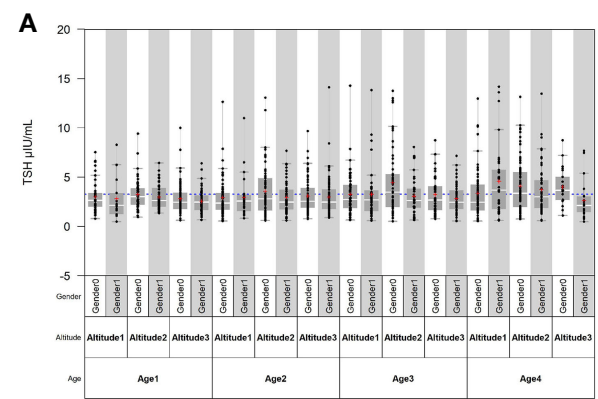

B
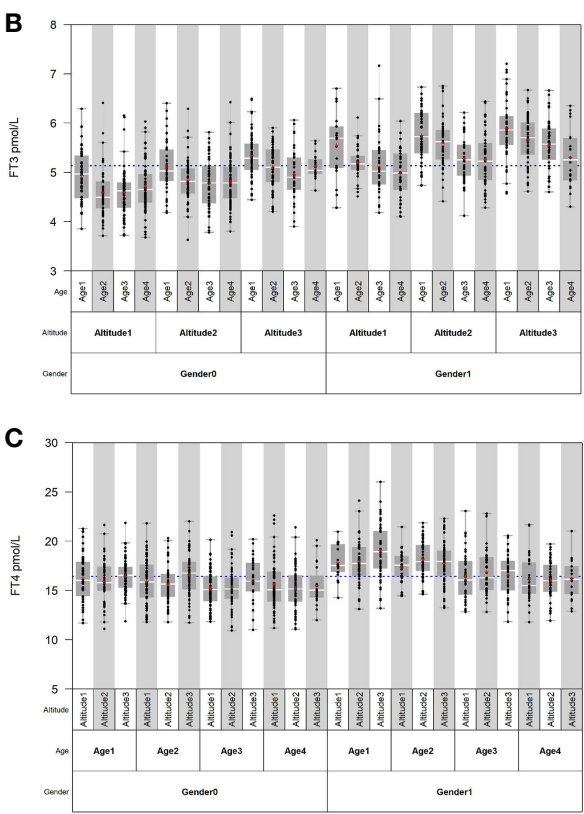

FIGURE 1 | Distribution of thyroid-related hormones in Tibetan population by sex, age, and altitude. (A-C) Distribution of TSH, FT3, and FT4 in Tibetan population by sex, age, and altitude.

and FT4 decreased with increasing age. Tibetans aged over 50 years have significant higher levels of TSH than those aged between 19 and 29 years $(P<0.001)$. FT3 increased with altitude $(P<0.001)$, while TSH and FT4 were less influenced by altitude. The results of multiple linear regression in Table 2 and the variance component analysis in Table $\mathbf{3}$ indicated that sex- and altitude-specific RIs for FT3 should be considered when the SDR $\geq 0.4$. The RIs for FT4 should be partitioned by sex with SDRsex of 0.4 .

\section{RIs of Thyroid-Associated Hormones for the Population at High Altitude}

The optimal parametrical models for RI establishment calculated by the refineR algorithm are shown in Figure 2 and Appendix 1. The specific results are shown in Table 4. RIs for FT3 were divided by sex and altitude, while RIs for FT4 were divided by sex. The RIs for FT3 and FT4 were overall higher in males than in females. RIs for FT3 elevated with increasing altitude as the RIs at altitudes III, II, and I were 4.09-4.98, 4.31-5.45, and 4.82-5.58 $\mathrm{pmol} / \mathrm{L}$ in females and $4.82-5.41,4.88-5.95$, and $5.26-6.06 \mathrm{pmol} /$ $\mathrm{L}$ in males. The total RIs for TSH, FT3, and FT4 were $0.764-$ $5.784 \mu \mathrm{IU} / \mathrm{ml}, 4.01-6.23 \mathrm{pmol} / \mathrm{L}$, and $12.19-20.70 \mathrm{pmol} / \mathrm{L}$, respectively. Compared with the RIs provided by the manufacturer, the lower and upper limits of the obtained RIs for TSH and FT4 were higher, and the RI for FT3 was narrower.

\section{DISCUSSION}

The current study utilized the refine $\mathrm{R}$ algorithm to formulate sex- and/or altitude-specific TSH, FT4 and FT3 RIs for indigenous Tibetans living at high altitude. Sex, age, and altitude had no effect on TSH levels in Tibetans and the RI for TSH was $0.764-5.784 \mu \mathrm{IU} / \mathrm{ml}$. The level of FT3 was influenced by both sex and altitude, with the upper and lower limits of the RIs being higher for males than females and both increasing with altitude. The limits of TSH as well as FT4 were found to be significantly higher in our study as compared with those provided by the manufacturer. A narrower RI range for FT3 was also found. Therefore, to avoid misdiagnosis or underdiagnosis of thyroid disease, it is recommended to apply the appropriate specific RI for the Tibetan population.

Thyroid-related hormones are closely related to the metabolism of the body, short-term stress, or long-term adaptation. Due to the particularity of the living environment, Tibetan people may have hormone levels inconsistent with those in the plain areas. The present study found little effects of altitude on serum TSH among Tibetans, which is consistent with the results of an observation study (11). Relatively short half-life of TSH (17 to $93 \mathrm{~min}$ ) (15), pulsatile secretion manners $(15,22)$ and feedback regulation of the HPT axis may roughly explain the phenomenon, although the exact mechanism is unclear. In euthyroid people, synthetic TSH is released in a manner combining a basal (non-pulsatile) and a pulsatile form and can be promptly cleared after action on the thyroid gland. The frequency, mass, and duration of a pulsatile can be rapidly adjusted to maintain the dynamic stability of TSH under the control of the HPT axis in response to environmental changes like hypobaric hypoxia, cold, and other harsh conditions on the plateau (10, 23). Meanwhile, the levels of thyroid-associated hormones were apparently elevated at higher altitude, with FT3 rising more obviously. Our results roughly concurred with previous studies which reported significant increases in thyroidassociated hormones for people following short-term or prolonged exposure to high-altitude environments $(11,17$, 24, 25). Elevated thyroid-associated hormones in high-altitude 


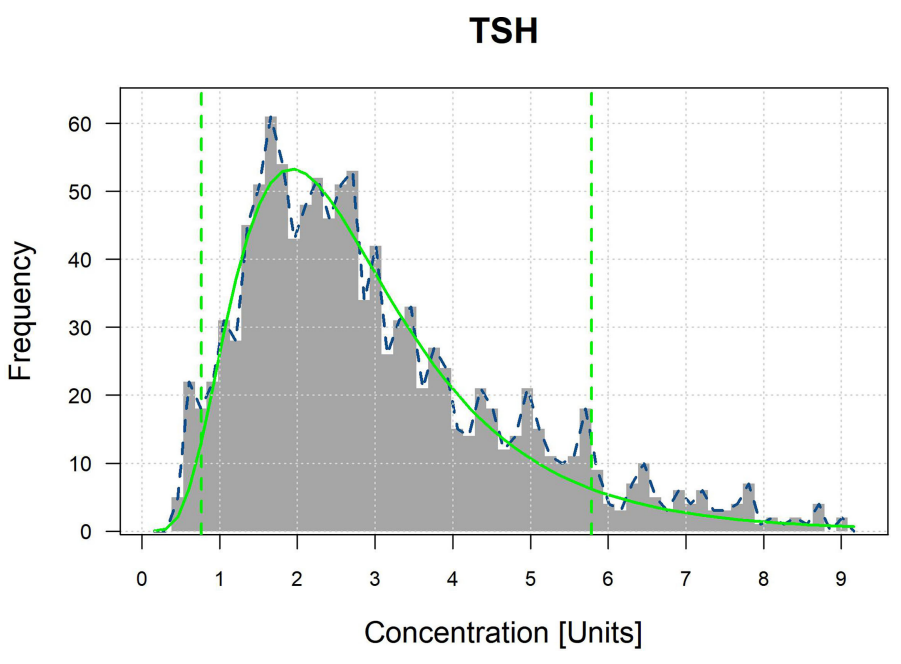

FIGURE 2 | Optimal parametrical models calculated by refineR algorithm for TSH.

TABLE 4 | Reference interval of thyroid-associated hormones.

\begin{tabular}{|c|c|c|c|c|c|c|c|c|}
\hline \multirow[t]{2}{*}{ Index } & \multirow[t]{2}{*}{ Units } & \multirow[t]{2}{*}{ Group } & \multicolumn{4}{|c|}{ refineR } & \multicolumn{2}{|c|}{ Manufacturer } \\
\hline & & & Lower limits of RI & $90 \% \mathrm{Cl}$ & Upper limits of RI & $90 \% \mathrm{Cl}$ & Lower limits of RI & Upper limits of RI \\
\hline $\mathrm{TSH}$ & $\mu \mathrm{U} / \mathrm{ml}$ & Total & 0.764 & $0.2276-0.9228$ & 5.784 & $4.0818-8.2030$ & 0.270 & 4.220 \\
\hline \multirow[t]{9}{*}{ FT3 } & $\mathrm{pmol} / \mathrm{L}$ & Total & 4.01 & 3.909-4.240 & 6.23 & 5.701-6.554 & 3.10 & 6.80 \\
\hline & & Female & 3.98 & $3.803-4.298$ & 5.79 & $5.370-6.077$ & & \\
\hline & & Altitude III & 4.09 & $3.777-4.424$ & 4.98 & $4.769-5.324$ & & \\
\hline & & Altitude II & 4.31 & $4.051-4.628$ & 5.45 & $5.119-5.762$ & & \\
\hline & & Altitude I & 4.82 & $4.411-5.048$ & 5.58 & $5.379-5.848$ & & \\
\hline & & Male & 4.58 & $4.141-5.088$ & 6.53 & $5.955-6.711$ & & \\
\hline & & Altitude III & 4.82 & $4.633-4.995$ & 5.41 & $5.260-5.590$ & & \\
\hline & & Altitude II & 4.88 & $4.480-5.244$ & 5.95 & $5.575-6.271$ & & \\
\hline & & Altitude I & 5.26 & 5.019-5.592 & 6.06 & $5.852-6.420$ & & \\
\hline \multirow[t]{3}{*}{ FT4 } & $\mathrm{pmol} / \mathrm{L}$ & Total & 12.19 & $11.417-13.599$ & 20.70 & $18.854-21.721$ & 12.00 & 20.00 \\
\hline & & Female & 12.36 & $11.422-14.316$ & 19.38 & $17.207-20.776$ & & \\
\hline & & Male & 14.84 & $12.407-16.318$ & 20.18 & $18.890-21.825$ & & \\
\hline
\end{tabular}

Altitude III, Nyingchi (altitude: 2,900 m); altitude II, Shigatse/Lhasa (altitude: 3,670-3,835 m); altitude I, Ali (altitude: 4,298-4,352 m).

environments facilitate the resistance of the body to the harsh environment of high altitude and seem to be independent on pituitary gland secretion of TSH $(24,26)$. Furthermore, altitudedependent FT3 rise may also be relevant to physiological changes caused by the relative lack of iodine, as studies have shown that Tibetan regions have lower urinary iodine levels and a higher risk of iodine deficiency compared with mainland China $(27,28)$. Mouse models also showed that iodine deficiency could induce changes in deiodinase activity in the thyroid or peripheral tissues to affect the conversion of T4 to T3 (29), and make monocarboxylate transporter 8 , one of the most important T3 relevant transporters, upregulated in the thyroid gland to transport more T3 into blood circulation (30).

Gender and age are important factors to be considered in the establishment of RIs for thyroid-related hormones. However, there is still a great controversy about whether the RIs should be partitioned by age $(7,12,14,31)$. In our study, the impact of age on RIs for TSH, FT3, and FT4 in Tibetans was modest. Although it was clear from the results that TSH levels were significant higher in the group aged over 50 years than in the group aged between 19 and 29 years, the RI could not be partitioned by age in our study and the RI for TSH was $0.764-5.784 \mu \mathrm{IU} / \mathrm{ml}$. Zhai et al., using the same instrument as we did, reported that the RIs for TSH in Chinese plain population $<65$ and $\geq 65$ years were $0.76-6.57$ and $0.75-8.86 \mathrm{mIU} / \mathrm{L}$, respectively (14). Difference in the upper limits may be explained by the age composition of the population living at different altitudes in China, as in the case of the three regions of Tibet, where the proportion of people aged $>65$ years was only approximately 5\% (32). Obvious discrepancies were observed for TSH, FT3, and FT4 levels between males and females, although the effect of sex on TSH level was not as remarkable as those on FT3 or FT4, which was 
consistent with previous studies $(14,27,33,34)$. Thus, sexspecific RIs for FT4 and FT3, but not for TSH, were proposed in our study and results showed that both the upper and lower limits of RIs for FT3 and FT4 were higher in males than in females, which can be interpreted by the potential effect of sex hormones on the HPT axis $(10,35)$.

At the end of this study, we compared the RIs calculated by the refineR algorithm with those proposed by the manufacturer. Higher upper and lower reference limits were found in Tibetans for TSH and FT4 (TSH: $0.764-5.784$ vs. $0.270-4.220 \mu \mathrm{IU} / \mathrm{ml}$, FT4: $12.19-20.70$ vs. $12.00-20.00 \mathrm{pmol} / \mathrm{L})$, as well as a narrower range for FT3 (4.01-6.23 vs. $3.10-6.80 \mathrm{pmol} / \mathrm{L})$. These discrepancies suggested that the direct use of the RIs of the manufacturer may lead to underdiagnosis of hyperthyroidism or overdiagnosis of subclinical hypothyroidism. The study highlights the importance of establishing appropriated RIs in high-altitude laboratories by an indirect method like the refineR algorithm. However, it is important that $90 \%$ CI of RIs show that the widths of CI of FT3 and FT4 are suitable. However, the $90 \%$ $\mathrm{CI}$ of the upper limit of reference interval for TSH is relatively wide, possibly due to the large variation of the TSH for populations at high altitude.

Our research has both advantages and disadvantages. Firstly, it is the first multicenter cross-sectional study to set up RIs for thyroid-related hormones in Tibetan population dwelling at the plateau, and the refineR algorithm was creatively used under this situation. Secondly, a detailed questionnaire was used to assist in screening subjects from Nyingchi, Shigatse, Lhasa, and Ali. Unequivocally, there are limitations in the current study, such as the lack of thyroid ultrasonography results and the utilization of only a single testing system. However, the refineR algorithm can help identify healthy individuals from a distribution mixed with a small proportion of pathological individuals and then obtain RIs according to the optimal parameter models. Considering that conventional definitions of apparent health, such as normal blood pressure and BMIs in the normal range, may not be applicable to high-altitude dwellers, the method in our study may be a preference to establish RIs. In addition, the RIs established for thyroid-related hormones may be only applicable to the Roche platform, as non-negligible discrepancy among assay methodologies has been revealed (13). Overall, we have currently established RIs for Tibetans living at high altitude for generations, and further studies should use real-world data to compare the prevalence of thyroid disease in populations dwelling at different altitudes for generations.

\section{CONCLUSION}

Altitude- and/or sex-specific RIs for thyroid-related hormones were established in Chinese people living at high-altitude areas based on the refineR algorithm. Significant differences have been found while comparing the obtained RIs with the RIs provided by the manufacturer. Therefore, establishment of specific RIs according to regional characteristics of Tibet should be recommended to avoid underdiagnosis or misdiagnosis of thyroid disease.

\section{DATA AVAILABILITY STATEMENT}

The original contributions presented in the study are included in the article/Supplementary Material Further inquiries can be directed to the corresponding author.

\section{ETHICS STATEMENT}

The studies involving human participants were reviewed and approved by the Ethics Committee of the People's Hospital of Tibet Autonomous Region (Approval No.ME-TBHP-2017-021) and the Ethics Committee of Peking Union Medical College Hospital (Approval No. S-K530). The patients/participants provided their written informed consent to participate in this study.

\section{AUTHOR CONTRIBUTIONS}

LQ designed the study. CM analyzed the data. CM, JZ, and YZ wrote this manuscript. $\mathrm{LH}, \mathrm{DW}$, and CM performed detection of the thyroid-related hormones. XC, LQ, and DW made suggestions for the revision of the manuscript. HL, JP, ZL, XL, $\mathrm{ZP}$, and LT helped in participant recruitment and sample collection. All authors reviewed the manuscript. All authors contributed to the article and approved the submitted version.

\section{FUNDING}

The work was supported by the Special Foundation Project for Human Resources Development of the Tibet Autonomous Region (2016000), Science and Technology Program of the Tibet Autonomous Region (2015XZ01G20), Beijing Key Clinical Specialty for Laboratory Medicine - Excellent Project (No. ZK201000) and Capital's Funds for Health Improvement and Research (CFH-2020-1-4014).

\section{SUPPLEMENTARY MATERIAL}

The Supplementary Material for this article can be found online at: https://www.frontiersin.org/articles/10.3389/fendo.2022. 816970/full\#supplementary-material 


\section{REFERENCES}

1. Taylor PN, Albrecht D, Scholz A, Gutierrez-Buey G, Lazarus JH, Dayan CM, et al. Global Epidemiology of Hyperthyroidism and Hypothyroidism. Nat Rev Endocrinol (2018) 14(5):301-16. doi: 10.1038/nrendo.2018.18

2. Li Y, Teng D, Ba J, Chen B, Du J, He L, et al. Efficacy and Safety of Long-Term Universal Salt Iodization on Thyroid Disorders: Epidemiological Evidence From 31 Provinces of Mainland China. Thyroid (2020) 30(4):568-79. doi: 10.1089/thy.2019.0067

3. Chaker L, Bianco AC, Jonklaas J, Peeters RP. Hypothyroidism. Lancet (2017) 390(10101):1550-62. doi: 10.1016/s0140-6736(17)30703-1

4. De Leo S, Lee SY, Braverman LE. Hyperthyroidism. Lancet (2016) 388 (10047):906-18. doi: 10.1016/s0140-6736(16)00278-6

5. Shan Z, Chen L, Lian X, Liu C, Shi B, Shi L, et al. Iodine Status and Prevalence of Thyroid Disorders After Introduction of Mandatory Universal Salt Iodization for 16 Years in China: A Cross-Sectional Study in 10 Cities. Thyroid (2016) 26(8):1125-30. doi: 10.1089/thy.2015.0613

6. Biondi B, Cappola AR, Cooper DS. Subclinical Hypothyroidism: A Review. JAMA (2019) 322(2):153-60. doi: 10.1001/jama.2019.9052

7. Mooijaart SP, Du Puy RS, Stott DJ, Kearney PM, Rodondi N, Westendorp RGJ, et al. Association Between Levothyroxine Treatment and ThyroidRelated Symptoms Among Adults Aged 80 Years and Older With Subclinical Hypothyroidism. JAMA (2019) 322(20):1977-86. doi: 10.1001/ jama.2019.17274

8. Dickens LT, Cifu AS, Cohen RN. Diagnosis and Management of Thyroid Disease During Pregnancy and the Postpartum Period. Jama (2019) 321 (19):1928-9. doi: 10.1001/jama.2019.5321

9. Feldt-Rasmussen U, Effraimidis G, Klose M. The Hypothalamus-PituitaryThyroid (HPT)-Axis and its Role in Physiology and Pathophysiology of Other Hypothalamus-Pituitary Functions. Mol Cell Endocrinol (2021) 525:111173. doi: 10.1016/j.mce.2021.111173

10. Keenan DM, Pichler Hefti J, Veldhuis JD, Von Wolff M. Regulation and Adaptation of Endocrine Axes at High Altitude. Am J Physiol Endocrinol Metab (2020) 318(2):E297-309. doi: 10.1152/ajpendo.00243. 2019

11. von Wolff M, Nakas CT, Tobler M, Merz TM, Hilty MP, Veldhuis JD, et al. Adrenal, Thyroid and Gonadal Axes are Affected at High Altitude. Endocr Connect (2018) 7(10):1081-9. doi: 10.1530/EC-18-0242

12. Cappola AR. The Thyrotropin Reference Range Should Be Changed in Older Patients. JAMA (2019) 322(20):1961-2. doi: 10.1001/jama.2019.14728

13. Kahapola-Arachchige KM, Hadlow N, Wardrop R, Lim EM, Walsh JP. AgeSpecific TSH Reference Ranges Have Minimal Impact on the Diagnosis of Thyroid Dysfunction. Clin Endocrinol (Oxf) (2012) 77(5):773-9. doi: 10.1111/ j.1365-2265.2012.04463.x

14. Zhai X, Zhang L, Chen L, Lian X, Liu C, Shi B, et al. An Age-Specific Serum Thyrotropin Reference Range for the Diagnosis of Thyroid Diseases in Older Adults: A Cross-Sectional Survey in China. Thyroid (2018) 28(12):1571-9. doi: $10.1089 /$ thy.2017.0715

15. van der Spoel E, Roelfsema F, van Heemst D. Within-Person Variation in Serum Thyrotropin Concentrations: Main Sources, Potential Underlying Biological Mechanisms, and Clinical Implications. Front Endocrinol (Lausanne) (2021) 12:619568. doi: 10.3389/fendo.2021.619568

16. Zhang J, Li W, Chen QB, Liu LY, Zhang W, Liu MY, et al. Establishment of Trimester-Specific Thyroid Stimulating Hormone and Free Thyroxine Reference Interval in Pregnant Chinese Women Using the Beckman Coulter UniCel ${ }^{\mathrm{TM}}$ DxI 600. Clin Chem Lab Med (2015) 53(9):1409-14. doi: 10.1515/cclm-2014-0615

17. Liu D, Li M, Wang Y, Jiang S, You L, Li F, et al. Effects of Moderate Altitude on Fasting Blood Glucose, Lipid Profile and Thyroid Hormones in Healthy Volunteers. Chin J Pathophysiol (2019) 35(09):1683-8. doi: 10.3969/j.issn. 1000-4718. 2019.09.023

18. Ma C, Wang X, Wu J, Cheng X, Xia L, Xue F, et al. Real-World Big-Data Studies in Laboratory Medicine: Current Status, Application, and Future Considerations. Clin Biochem (2020) 84:21-30. doi: 10.1016/j.clinbiochem. 2020.06.014

19. Jones GRD, Haeckel R, Loh TP, Sikaris K, Streichert T, Katayev A, et al. Indirect Methods for Reference Interval Determination - Review and
Recommendations. Clin Chem Lab Med (2018) 57(1):20-9. doi: 10.1515/ cclm-2018-0073

20. Ma C, Cheng X, Xue F, Li X, Yin Y, Wu J, et al. Validation of an Approach Using Only Patient Big Data From Clinical Laboratories to Establish Reference Intervals for Thyroid Hormones Based on Data Mining. Clin Biochem (2020) 80:25-30. doi: 10.1016/j.clinbiochem.2020.03.012

21. Ammer T, Schützenmeister A, Prokosch HU, Rauh M, Rank CM, Zierk J. Refiner: A Novel Algorithm for Reference Interval Estimation From RealWorld Data. Sci Rep (2021) 11(1):16023. doi: 10.1038/s41598-021-95301-2

22. Roelfsema F, Boelen A, Kalsbeek A, Fliers E. Regulatory Aspects of the Human Hypothalamus-Pituitary-Thyroid Axis. Best Pract Res Clin Endocrinol Metab (2017) 31(5):487-503. doi: 10.1016/j.beem.2017.09.004

23. Mendoza A, Hollenberg AN. New Insights Into Thyroid Hormone Action. Pharmacol Ther (2017) 173:135-45. doi: 10.1016/j.pharmthera.2017.02.012

24. Basu M, Pal K, Malhotra AS, Prasad R, Sawhney RC. Free and Total Thyroid Hormones in Humans at Extreme Altitude. Int J Biometeorol (1995) 39(1):1721. doi: $10.1007 / \mathrm{bf} 01320888$

25. Richalet JP, Letournel M, Souberbielle JC. Effects of High-Altitude Hypoxia on the Hormonal Response to Hypothalamic Factors. Am J Physiol Regul Integr Comp Physiol (2010) 299(6):R1685-92. doi: 10.1152/ajpregu.00484.2010

26. Nepal O, Pokhrel BR, Khanal K, Gyawali P, Malik SL, Koju R, et al. Thyroid Hormone Levels in Highlanders- a Comparison Between Residents of Two Altitudes in Nepal. Kathmandu Univ Med J (KUMJ) (2013) 11(41):18-21. doi: 10.3126/kumj.v11i1.11017

27. Ning P, Ren Q, Teng D, Zhang Z, Lv X, Meng S, et al. Current Iodine Nutrition Status and Prevalence of Thyroid Disorders in Tibetan Adults in an Oxygen-Deficient Plateau, Tibet, China: A Population-Based Study. Thyroid (2020) 30(5):759-66. doi: 10.1089/thy.2019.0669

28. Zou Y, Li H, Pang J, Liu X, Zejipuchi, Tian L, et al. An Evaluation of Urine and Serum Iodine Status in the Population of Tibet, China: No Longer an Iodine-Deficient Region. Nutrition (2021) 82:111033. doi: 10.1016/j.nut.2020. 111033

29. Lavado-Autric R, Calvo RM, de Mena RM, de Escobar GM, Obregon MJ. Deiodinase Activities in Thyroids and Tissues of Iodine-Deficient Female Rats. Endocrinology (2013) 154(1):529-36. doi: 10.1210/en.2012-1727

30. Hu Z, Zhuo X, Shi Y, Liu X, Yuan J, Li L, et al. Iodine Deficiency Up-Regulates Monocarboxylate Transporter 8 Expression of Mouse Thyroid Gland. Chin Med J (Engl) (2014) 127(23):4071-6. doi: 10.3760/cma.j.issn.03666999.20141314

31. Carle A, Pedersen IB, Knudsen N, Perrild H, Ovesen L, Andersen S, et al. Hypothyroid Symptoms Fail to Predict Thyroid Insufficiency in Old People: A Population-Based Case-Control Study. Am J Med (2016) 129(10):1082-92. doi: 10.1016/j.amjmed.2016.06.013

32. Liao G, Liu Z. A Study on Situation and Characteristics of the Age Structure of Tibet Population : Based on the Analysis of the Sixth Census Data. Northwest Population J (2014) 35(02):13-6. doi: 10.15884/j.cnki.issn.1007-0672.2014. 02.008

33. Li ZZ, Yu BZ, Wang JL, Yang Q, Ming J, Tang YR. Reference Intervals for Thyroid-Stimulating Hormone and Thyroid Hormones Using the Access TSH 3rd IS Method in China. J Clin Lab Anal (2020) 34(5):e23197. doi: $10.1002 /$ jcla. 23197

34. Zou Y, Wang D, Cheng X, Ma C, Lin S, Hu Y, et al. Reference Intervals for Thyroid-Associated Hormones and the Prevalence of Thyroid Diseases in the Chinese Population. Ann Lab Med (2021) 41(1):77-85. doi: 10.3343/alm.2021. 41.1.77

35. Hadlow NC, Rothacker KM, Wardrop R, Brown SJ, Lim EM, Walsh JP. The Relationship Between TSH and Free $\mathrm{T}_{4}$ in a Large Population is Complex and Nonlinear and Differs by Age and Sex. J Clin Endocrinol Metab (2013) 98 (7):2936-43. doi: 10.1210/jc.2012-4223

Conflict of Interest: The authors declare that the research was conducted in the absence of any commercial or financial relationships that could be construed as a potential conflict of interest.

Publisher's Note: All claims expressed in this article are solely those of the authors and do not necessarily represent those of their affiliated organizations, or those of the publisher, the editors and the reviewers. Any product that may be evaluated in 
this article, or claim that may be made by its manufacturer, is not guaranteed or endorsed by the publisher.

Copyright (๑) 2022 Ma, Zhong, Zou, Liu, Li, Pang, Liu, Zejipuchi, Tian, Hou, Wang, Cheng and Qiu. This is an open-access article distributed under the terms of the
Creative Commons Attribution License (CC BY). The use, distribution or reproduction in other forums is permitted, provided the original author(s) and the copyright owner(s) are credited and that the original publication in this journal is cited, in accordance with accepted academic practice. No use, distribution or reproduction is permitted which does not comply with these terms. 